\title{
El síndrome de Lemierre: Una patología resurgente. A propósito de una observación pediátrica
}

\section{Lemierre's syndrome: A resurgent pathology about a pediatric observation}

\author{
O Ibsaine ${ }^{1}$, K Aitidir², H Berrah¹, Z Arrada¹.
}

\begin{abstract}
RESUMEN
El síndrome de Lemierre (SL) es una emergencia diagnóstica y requiere una terapéutica urgente. Se define como una tromboflebitis séptica de la vena yugular interna (VYI) secundaria a una infección orofaríngea. En la mayoría de los casos el germen implicado es el Fusobacterium necrophorum (FN). Afecta tanto al adulto joven como al adolescente pero muy poco al niño. Esta rara afección debe beneficiarse de un diagnóstico precoz. En el caso contrario, se acompaña con un riesgo elevado de mortalidad.

Les exponemos en este trabajo, el caso de una niña de 6 años con un SL tras una otitis media aguda asociada a manifestaciones cutáneas de la septicemia. La paciente mejoró bajo una combinación de antibióticos (8 semanas) y anticoagulantes (3 meses).

Palabras clave: Síndrome de Lemierre, tromboflebitis, Fusobacterium necrophorum.
\end{abstract}

\begin{abstract}
Lemierre's syndrome is a rare, acute and severe entity. It is characterized by thrombosis of the internal jugular vein and metastatic infections especially lung localization. The main pathogen is Fusobacterium necrophorum. This pathology concerns rarely the child. Early diagnosis is crucial otherwise the mortality will increase. We report of Lemierre's syndrome in a girl of 6 years old with favourable outcome. Antibiotherapy targeting anaerobes has been rapidly Introduced and maintained 8 weeks. The anticoagulation has been maintained 3 months.
\end{abstract}

Keys words: Lemierre's syndrome, Thrombophlebitis, Fusobacterium necrophorum.

\footnotetext{
1 Servicio de Pediatría B. Centro Hospital Universitario Nafissa Hamoud Hussein-Dey, Argel.

2 Centro Especializado Militar en Consultaciones Hussein-dey, Argel.
} 


\section{INTRODUCCIÓN:}

El síndrome de Lemierre (SL) consiste en una trombosis séptica de venayugular interna (VYI). En $80 \%$ de los casos, resulta de una infección orofaríngea, con manifestaciones a distancia, sobre todo pulmonares. Generalmente se incrimina el Fusobacterium necrophorum (FN) 1 .

Esta enfermedad se encuentra más en los adultos jóvenes y los adolescentes, afecta raramente al niño $0^{2,3}$. El desconocimiento de la afección retrasa el diagnóstico y como consecuencia, en una agravación del pronóstico aumentando así el riesgo de mortalidad.

\section{CASO CLÍNICO}

Se trata de L. Anfel, una niña de 6 años que fue hospitalizada por fiebre. En sus antecedentes se anota un colesteatoma.

Su historia clínica se caracterizó por una fiebre resistente a los antipiréticos desde 20 días. Fue tratada por otitis media aguda (OMA). Recibió una asociación amoxicilina-clavulánico durante 7 días, después cefixima por 10 días. Un día antes de su admisión, la sintomatología se completó con cefaleas intensas y mareo fuerte.

En su examen manifestó una alteración del estado general con fiebre de $39^{\circ} \mathrm{C}$, un dolor de tortícolis en el lado izquierdo, una OMA izquierda con secreciones purulentas y dolor a la presión de la región retroauricular homolateral. No hay desprendimiento del pabellón de la oreja. El examen neurológico muestra un síndrome meníngeo.

Este cuadro clínico motiva la práctica de una exploración urgente. Se destacan un síndrome inflamatorio con PCR >48 mg/l, fibrinógeno 5,26 g/l, una hiperleucositosis $11.200 / \mathrm{ml}$ con desviación izquierda. El resto de los parámetros infecciosos son normales incluyendo la punción lumbar. Las funciones renales y hepáticas no presentan anomalías.

Se realizan TC cervical y cerebral con contraste y una resonancia que muestran (Figuras 1, 2, 3):

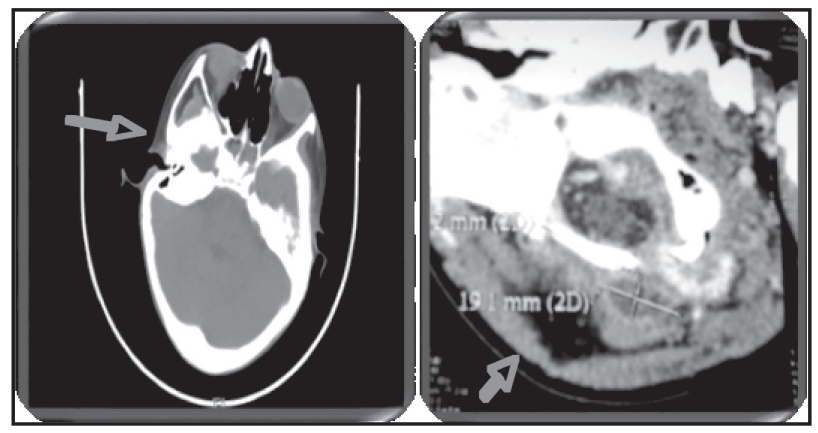

Figura 1. Aspecto de ocupación mastoidea sin lesiones óseas (a la izquierda). Figura 2. Absceso del tejido blando cervical izquierdo (a la derecha).

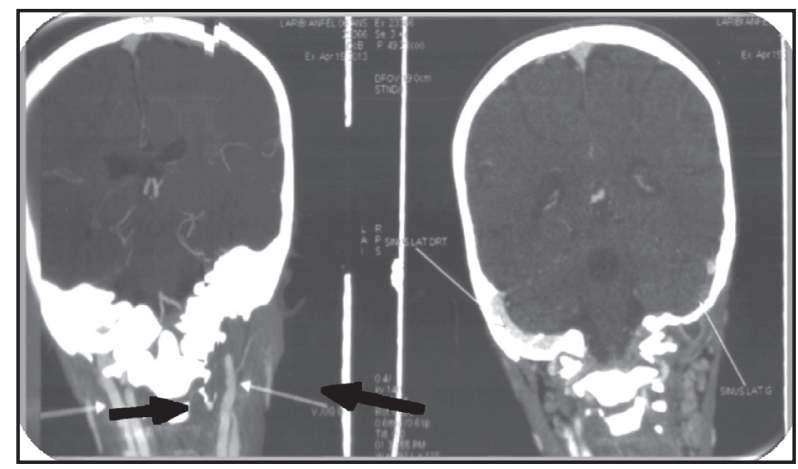

Figura 3. Trombosis y oclusión de la VYI izquierda. 
una tromboflebitis extendida de la VYI izquierda hasta el seno lateral izquierdo. Una mastoiditis y varios abscesos cervicales, el más voluminoso mide $19 \times 11 \mathrm{~mm}$.

La radiografía del tórax y la ecografía del abdomen son normales.

El análisis de la hemostasia (factores de coagulación: proteínas S, C, antitrombina III), anticuerpos antifosfolípidos son sin particularidad. Las serologías de CMV, EBV son negativas eliminando una reciente infección. Sin embargo, el estudio bacteriológico -hecho tras la toma de cefixima- no encuentra ninguna bacteria ni en los hemocultivos ni en el pus auricular.

El diagnóstico del SL es establecido por la asociación de: Fiebre secundaria a una OMA, tromboflebitis de la VYI izquierda y absceso cutáneos cervicales.

Se administran antibióticos endovenosos con actividad frente a estreptococos, estafilococos aureus y anaerobios durante 15 días con la vancomicina, mientras que el metronidazol y la cefotaxima han sido combinados y mantenidos durante 4 semanas. Simultáneamente, se asocia, una heparina de alto peso molecular por 5 días.

La evolución es favorable con una apirexia, regresión y luego disminución de las cefaleas y de la tortícolis, así como de la otitis. Se normalizan las alteraciones analíticas. La TAC de control realizada tras 30 días de tratamiento es satisfactoria con desaparición de abscesos cutáneos (AC), pero con persistencia de la trombosis de la VYI por lo que se siguen dando los antibióticos (ATB) y se administra oralmente una antivitamina $\mathrm{K}$ como anticoagulante (ACG), por duraciones prolongadas respectivamente de uno y tres meses. Se obtiene la repermeabilización de la VYI al final de la terapéutica.

\section{DISCUSIÓN}

El SL es un estado séptico raro y grave. Fue descrito por primera vez en 1936 por el microbiólogo André Lemierre quien publicó una serie de 20 casos que presentaron una septicemia severa consecuencia de una infección orofaríngea causada por un germen anaerobio y complicada de una tromboflebitis (TFB) de la VYI asociada a émbolos sépticos (ES)1.
Afecta más al adolescente y al adulto joven inmunocompetentes con predominio masculino ${ }^{2,3}$. Sin embargo, en la literatura aparecen casos de niños sanos afectados por el SL, el más joven citado es un lactante de 2 meses $^{4}$. Según los estudios, en la edad pediátrica, esta patología se relaciona sobre todo con una OMA ${ }^{5,4,6}$, también una sinusitis, una mastoiditis, un absceso dental $33,7,5,5$ incluso una parotiditis ${ }^{9}$ pueden ser el punto inicial del desarrollo del SL. En el hospital pediátrico de Wisconsin (1995-2002) sobre 14 casos, todos han presentado angina ${ }^{10}$.

Gracias a los antibióticos y a la higiene oral y dental, el SL ha resultado rarísimo en las décadas 60 y 70 llegando a ser nominado «enfermedad olvidada» ${ }^{2,8}$. En contraparte, Hagelskjaer de Dinamarca, ha notado un aumento de su incidencia anual, pasando de 0,8 casos/millón (1990-1995) a 3,6 casos/millón (1998-2001)³. Esto podría explicarse por muchas razones:

- Tratamientos ATB reservados sólo para las faringitis con cultivos positivos a estreptococos grupo A.

- El uso con más frecuencia de algunos antibióticos que se administran una 0 dos veces al día (macrólidos), pero son inactivos sobre los anaerobios.

- Un aumento del uso de los antiinflamatorios en el tratamiento de las anginas.

- La aparición de resistencia a los ATB.

La prevalencia de los gérmenes anaerobios encontrados en caso de una otitis oscila entre $12 \%-25 \%{ }^{11-12}$. En cuanto a la principal etiología, a menudo se trata del FN, anaerobio Gram negativo, inmóvil, que forma parte de la flora del orofarinx, del tracto respiratorio superior y también del aparato genital femenino $0^{13}$. Debe su virulencia a las endotoxinas elaboradas como la hemolisina que favorece la anaerobiosis 0 a la leucocidina alterando la inmigración de los polinucleares 0 a la hemaglutinina, provocando una agregación de las plaquetas lo que lleva a una coagulación vascular diseminada y trombosis ${ }^{14}$.

Los hemocultivos y el cultivo del líquido del oído positivo son elementos claves para confirmar la sepsis, su negatividad no elimina el diagnóstico siempre que el cuadro clínico sea sugerente, como ocurre en nuestro caso. De otra parte, el laboratorio no recurre sistemáticamente a los métodos específicos para la búsqueda de los gérmenes 
anaerobios sobre todo que el aislamiento de FN es difícill ${ }^{14}$ y puede tener algunos días de incubación. En consecuencia, ante toda infección orofaríngea resistente a los antibióticos, se debe pensar en SL y conducir entonces, a realizar pruebas adecuadas. Otros microrganismos tales como el $F$ nucleatum, el $F$ mortiferum, el estreptococos constelletus, el Haemophilus influenza y el estreptococos del grupo $\mathrm{A}$ han sido identificados como responsables de la génesis del SL ${ }^{15}$. También existen coinfecciones con gérmenes aerobios. En 10\% de los casos, ninguna bacteria ha sido aislada ${ }^{2}$. En un estudio de Ramírez se encontró el citomegalovirus y el Epstein Barr virus ${ }^{16}$ cuyas serologías correspondientes son negativas en nuestra paciente.

Es probable que la disminución de las defensas, consecutiva a una faringitis viral o bacteriana, favorezca la multiplicación del germen anaeróbico, invadiendo así la mucosa, provocando abscesos. EI SL está caracterizado por una infección de la esfera ORL (angina, laringitis, faringitis, absceso dental...), evolucionando en un flegmón luego una TFB séptica de VYI homolateral a través de una difusión local o por el intermedio de venas o vasos linfáticos y al final diseminación por vía hematógena y linfática en los 4 a 8 días siguientes. En nuestra observación, es posible que la inflamación y la infección del oído medio, se hayan propagado al periostio mastoideo provocando una mastoiditis y después por contigüidad, al seno lateral causando TFB extendiéndose hasta cerca la VYI ${ }^{17}$. Luego, Ios ES pueden migrar a distancia, afectando más frecuentemente el pulmón, pero también el bazo, el hígado, el corazón, las articulaciones y los tejidos blandos ${ }^{18,19}$.

Por el hecho de estar a menudo silenciosa e insidiosa, la trombosis de la VYI debe beneficiarse de una exploración radiológica completa. Se puede recurrir al doppler, pero es superada por RM o TAC con inyección para una mayor certeza diagnóstica. El doppler se utiliza más en busca de abscesos hepáticos 0 esplénicos.

La terapéutica médica está basada en una antibioterapia endovenosa dirigida contra los anaerobios y aplicada de manera prolongada. No hay consenso bien definido. Generalmente, FN es sensible a la penicilina, clindamicina, metronidazol, cefalosporinas, cloranfenicol ${ }^{4}$. Clásicamente, se administra una biterapia asociando una penicilina a alta dosis y metronidazol durante 15 días por vía endovenosa, sustituida por amoxicilina-ácido clavulánico oral por 4 semanas ${ }^{5,9}$. No obstante, ante la aparición de bacterias resistentes que producen betalactamasa se utilizarían otros principios activos tales como piperacilina, imipenem, clindamicina ${ }^{20}$; también esta última se usa en el caso de una alergia a la penicilina ${ }^{4}$. Algunos autores como Le Monnier y col recomiendan el uso sistemático de ATB con espectro anaeróbico en el tratamiento de angina u otitis a fin de evitar complicaciones ${ }^{21}$.

Respecto a la anticoagulación, los estudios son controvertidos. Para algunos, hay que restringir su uso, porque esto representa una fuente de diseminación de la infección a través de los ES disueltos. Otros estiman su efecto beneficioso sobre TFB, limitando así su extensión ${ }^{22}$. La evolución es globalmente favorable. Sin embargo, se ha observado una persistencia de la trombosis de VYI hasta 6 meses en $44 \%$ de los casos $^{23}$, como fue anotado en nuestra observación.

\section{CONCLUSIÓN}

El SL en pediatría es una afección desconocida y rara. El diagnóstico es sobre todo clínico. Se debe evocar ante toda infección orofaríngea resistente y asociada a una sepsis o a dolor latero cervical. La exploración radiológica en búsqueda de una trombosis de la VYI y el cultivo bacteriológico permitirán afirmar el diagnóstico. El tratamiento antibiótico bien adaptado no debe sufrir de ningún retraso a fin de preservar un buen pronóstico.

\section{BIBLIOGRAFÍA}

1. Lemierre A. On certain septicemias due to anaerobic organisms. Lancet 1936; 227: 7013.

2. Hagelskjaer lH, Prag J, Malczynski J, Kristensen $\mathrm{JH}$. Incidence and clinical epidemiology of necrobacillosis, including Lemierre's syndrome, in Denmark 1990-1995. Eur J Clin Microbiol Infect Dis 1998; 17: 561-5.

3. Hagelskjaer Kristensen L, Prag J. Lemierre's syndrome and other disseminated Fusobacterium necrophorum infections in Denmark: a 
prospective epidemiological and clinical survey. Eur J Clin Microbiol Infect Dis 2008; 27: 77989.

4. LitTerio MR, Soto AE, Aguirre CB, et al. Lemierre's syndrome: case report in a paediatric patient. Anaerobe 2004; 10: 151-4.

5. Passalidou P, Berlioz M, Ballly C, Boutte P. Syndrome de Lemierre: une infection oropharyngée compliquée. Arch Pédiatr 2008; 15: 1775-17.

6. Valla F, Berchiche C, Floret D. Nécrobacillose et syndrome de Lemierre : à propos d'un cas Necrobacillosis and Lemierre's syndrome. Arch Pédiatr 2003; 10: 1068-70.

7. Blaise S, Colombe B, Millet C, Poulain C, Bosseray A, Carpentier PH, et al. Jugular thrombosis with fever: what about Lemierre syndrome? J Mal Vasc 2005; 30: 231-2.

8. McMullan R, McConville C, Clarke JC, et al. Lemierre syndrome: remember the forgotten disease. Ulster Med J 2004; 73: 123-5.

9. Valleix B, Floccard B, Hautin E, Faure F, Allaouchiche B. A propos d'un syndrome de Lemierre secondaire à une parotidite. Ann Fr Anesth Réanim 2011; 30: 692-5.

10. Goldenberg NA, Knapp-Clevenger R, Hays T, et AL. Lemierre's and Lemierre's-like syndromes in children: survival and thromboembolic outcomes. Pediatrics 2005; 116: 543-8.

11. Brook I. The role of anaerobic bacteria in otitis media: microbiology, pathogenesis, and implications on therapy. Am J Otolaryngol 1987; 8: 109-17.

12. BROoK I, BuRKE P. The management of acute, serous and chronic otitis media; the role of anaerobic bacteria. J Hosp Infect 1992; 22: 75-87.

13. Hagelskjaer Kristensen L, Prag J. Human necrobacillosis, with emphasis on Lemierre's syndrome. Clin Infect Dis 2000; 31: 524-32.

14. TanZL, Nagaraua TG, Chengappa MM. Fusobacterium necrophorum infections: virulence factors, pathogenic mechanism and control measures. Vet Res Commun 1996; 20: 113-40.

15. Blumberg D, BiAnchett। MG. Lemierre syndrome caused by group A streptococci. Pediatr Infect Dis J 2007; 26: 1075.

16. Ramírez S, Hild TG, Rudolph CN, et al. Increased diagnosis of Lemierre syndrome and other Fusobacterium necrophorum infections at a children's hospital. Pediatrics 2003; 112: e380.

17. Françoıs M. Complications des otites moyennes aiguës et chroniques. EMC-Oto- rhinolaryngologie 2005; 2: 92-106.

18. Courtin P, Toro A, Gazagnes M, Berrouba A, Gallardo M, Dembele A. Syndrome de Lemierre. Ann Fr Anesth Reanim 2010; 29: 799-802.

19. Cirus Schahab. Le syndrome de Lemierre, une affection en partie «oubliée», mais qui n'a pas disparu. Forum Med Suisse 2012; 12 (19): 386-8.

20. Ridgway JM, Parikh DA, Wright R, Holden P, Armstrong W, Camilon F, et al. Lemierre syndrome: a pediatric case series and review of literature. Am J Otolaryngol 2010; 31: 38-45.

21. Le Monnier A, Jamet A, Carbonnelle E, et AL. Fusobacterium necrophorum middle ear infections in children and related complications: report of 25 cases and literature review. Pediatr Infect Dis J 2008; 27: 613-7.

22. Courtin P, Toro A, Gazagnes M, et al. Syndrome de Lemierre. Ann Fr Anesth Reanim 2010; 29 : 799-802.

23. Goldenberg NA, Knapp-Clevenger R, Hays T, et AL. Lemierre's and Lemierre's-like syndromes in children: survival and thromboembolic outcomes. Pediatrics 2005; 116: 543-8. 